ISSN 0103-5150

Fisioter. Mov., Curitiba, v. 25, n. 3, p. 551-559, jul./set. 2012

Licenciado sob uma Licença Creative Commons

\title{
Efeitos da dominância unilateral dos membros inferiores na flexibilidade e no desempenho isocinético em mulheres saudáveis
}

\author{
Effects of unilateral dominance of the lower limbs on \\ flexibility and isokinetic performance in healthy females
}

\author{
Gabriel Peixoto Leão Almeida ${ }^{[a]}$, Kysia Karine Almeida Carneiro ${ }^{[b]}$, \\ Heleno Carneiro Rolim de Morais ${ }^{[\mathrm{c}]}$, Júlia Barreto Bastos de Oliveira ${ }^{[\mathrm{d}]}$
}

[a] Fisioterapeuta, especializando em Fisioterapia no Esporte pelo Centro de Traumatologia do Esporte (CETE), do Departamento de Ortopedia e Traumatologia da Universidade Federal de São Paulo (DOT/Unifesp), São Paulo, SP - Brasil, e-mail: gabriel_alm@hotmail.com

[b] Fisioterapeuta, especializanda em Fisioterapia Traumato-ortopédica Funcional pela Universidade Nove de Julho (Uninove), São Paulo, SP - Brasil, e-mail: kysiakarine@hotmail.com

[c] Fisioterapeuta, especialista em Educação em Saúde Pública, professor e coordenador do Programa de Dinamometria Isocinética (PDI) do Departamento de Fisioterapia da Universidade de Fortaleza (Unifor), Fortaleza, CE - Brasil, e-mail helenohcrm@unifor.br

[d] Fisioterapeuta, mestre em Distúrbio da Comunicação Humana pela Universidade Federal de São Paulo (Unifesp), professora do Departamento de Fisioterapia da Universidade de Fortaleza (Unifor), Fortaleza, CE - Brasil, e-mail: juliabbo@terra.com.br

\section{Resumo}

Introdução: A dominância unilateral dos membros inferiores pode causar desequilíbrios entre os grupos musculares contralaterais, predispondo ambas as pernas a lesões. Assim, o conhecimento de deficits comparativos unilaterais é importante nas medidas de prevenção e avaliação do paciente. Objetivo: Verificar a influência das atividades diárias na diferença dos membros dominante (MD) e não dominante (MND) quanto a flexibilidade, Pico de Torque (PT), Trabalho Máximo (TM), Potência Máxima (PM) dos flexores e extensores do joelho. Métodos: A amostra foi constituída por 23 mulheres saudáveis e não praticantes de atividade física. A flexibilidade foi avaliada pelo Teste do Ângulo Poplíteo (TAP) para isquiostibiais e Teste de Thomas (TT) para quadríceps-femoral; a avaliação isocinética foi realizada no modo concêntrico para extensão e flexão do joelho. Para o PT e o TM, foram realizadas cinco repetições na velocidade angular 
de $60^{\circ}$ /seg e, para PM, 15 repetições em $240^{\circ}$ /seg. Todas as avaliações foram realizadas pelo mesmo pesquisador. Resultados: Não houve diferença estatisticamente significante quanto à flexibilidade obtida pelo TAP e pelo TT $(\mathrm{p}>0,05)$. Porém, em todas as variáveis isocinéticas avaliadas houve uma diferença significativamente relevante entre o MD e o MND $(\mathrm{p}<0,01)$, com média do índice de simetria dos membros superior a $10 \%$. Conclusão: De acordo com os dados obtidos, as atividades do cotidiano em mulheres saudáveis causam diferenças entre o MD e o MND, sendo constatadas pela avaliação isocinética quanto a PT, TM e PM; porém, tais diferenças não foram visualizadas quanto à flexibilidade.

Palavras-chave: Amplitude de movimento articular. Avaliação de desempenho. Extremidade inferior.

\section{Abstract}

Introduction: The unilateral dominance of the lower limbs can cause asymmetries between the contralateral muscle groups, predisposing both legs to possible injury. Thus, knowledge of unilateral deficits is important in the prevention and outcomes. Objective: To assess the difference of daily activities on the dominant (DL) and non-dominant (NDL) limb as flexibility, peak torque (PT), maximum work (MW), maximum power (MP) for flexors and extensors of the knee. Method: 23 healthy women without symptoms. Flexibility was assessed using the popliteal angle test (PAT) for hamstring and Thomas test (TT) for quadriceps, the isokinetic assessment was carried out for the concentric extension and flexion of the knee. For the PT and MW were performed five repetitions at an angular velocity of 60o/sec, and for MP, 15 repetitions at 240o/sec. All evaluations were performed by the same researcher. Results: No statistically significant difference as the bilateral flexibility through the TAP and TT ( $p>0.05)$. However, all the isokinetic variables were assessed with a significant difference between the DL and NDL $(p<0.01)$, with an average of limb symmetry index exceeding $10 \%$. Conclusion: According to the data, the daily activities in healthy women cause differences between the DL and NDL, as evidenced by the isokinetic evaluation as to the PT, MW and MP; however, these differences were not viewed as flexibility.

Keywords: Range of motion. Employee performance appraisal. Lower extremity.

\section{Introdução}

O conceito popular de dominância parte da característica de preferência de um membro na execução de tarefas que exijam força, equilíbrio, coordenação e propriocepção, sendo que o membro não dominante (MND) favorece a estabilização necessária para a execução dos movimentos do indivíduo (1). Essa assimetria motora é uma consequência da lateralidade humana, explicada pela ideia de que os dois hemisférios cerebrais são funcionalmente desiguais, expressando-se em demandas diferentes para os membros (2-4).

A assimetria dos membros inferiores pode levar a alterações biomecânicas de movimento e postura, afetando negativamente ambos os membros (5-7). A maior demanda em relação ao membro dominante (MD) aumenta o estresse sobre as articulações dessa extremidade, e o excesso de confiança também pode resultar em fraqueza no membro contralateral, o que diminui a capacidade do MND para absorver grandes forças associadas a atividades diárias e esportivas $(6,8,9)$.

Durante a reabilitação, a simetria de força dos membros é avaliada e utilizada como um dos critérios de evolução para determinar o nível de participação do indivíduo em atividades desportivas e da vida diária. Amplitude de movimento, força muscular, resistência e potência também são frequentemente mensuradas para avaliar a simetria dos membros $(10$, 11). Por isso, é importante determinar se os efeitos da dominância unilateral podem gerar diferenças de performance entre os membros.

Não existe, na literatura, um consenso sobre como determinar a dominância de cada indivíduo. As pesquisas trazem vários parâmetros que podem definir qual é o membro dominante, como: um simples chute na bola (12); teste de subir escada (13); single hop test em distância (14); e autorrelato dos indivíduos (15).

A maioria dos estudos comparando os membros por meio do dinamômetro isocinético é realizada em atletas, principalmente em jogadores de futebol $(5,6$, 
$15,16)$. Por isso, existe a necessidade de pesquisas em indivíduos não praticantes de atividade física, tornando possível elucidar os efeitos apenas das atividades diárias na dominância unilateral.

Diante do exposto, o objetivo desse estudo foi verificar as diferenças entre o MD e o MND, em mulheres saudáveis, assintomáticas e não praticantes de atividade física, quanto a: flexibilidade, por meio do Teste do Ângulo Poplíteo (TAP) e do Teste de Thomas (TAP), pico de torque (PT), trabalho máximo (TM), potência máxima (PM) dos extensores e flexores do joelho, avaliados pelo dinamômetro isocinético.

\section{Materiais e métodos}

\section{Sujeitos}

Participaram da pesquisa 23 indivíduos do sexo feminino, com idade média de $22,6 \pm 1,8$ anos, peso de $56,3 \pm 6,3 \mathrm{~kg}$, altura de $1,59 \pm 0,05 \mathrm{~m}$ e IMC de 22 $\pm 2,4 \mathrm{~kg} / \mathrm{m}^{2}$. Como critério de inclusão, foi preconizado que deveriam ter entre 18 e 30 anos e história de, no mínimo, três meses sem realizar qualquer atividade física. Foram excluídas as que tivessem história recente de: lesão nos membros inferiores, contraindicação de acordo com o formulário de Par-Q (17), realização de atividade física no período da pesquisa, IMC acima de 28 , dores na coluna lombar, história de lesão prévia no joelho e/ou no quadril. Para determinar o membro dominante, as participantes imaginavam uma bola à sua frente $\mathrm{e}$ era feito o seguinte questionamento: "Com que perna você chutaria essa bola?" $(10,18)$.

Este estudo foi inicialmente aprovado pelo Comitê de Ética em Pesquisa em Seres Humanos da Universidade de Fortaleza (Coética), com o protocolo $\mathrm{n}^{\circ} 337 / 08$, e todos os participantes foram esclarecidos sobre os procedimentos a que seriam submetidos, tendo assinado um "Termo de Consentimento Livre e Esclarecido".

\section{Procedimentos}

Para execução da pesquisa, foi utilizado um goniômetro universal da marca $\operatorname{Carci}^{\circledR}$, um dinamômetro isocinético Cybex ${ }^{\circledR}$ (1200), uma maca ISP ${ }^{\circledR}$, uma balança Filizola ${ }^{\circledR}$ e uma faixa para estabilização das avaliações.
Na avaliação do encurtamento muscular, foram marcados três pontos de referência para as aferições goniométricas: entre o sulco do bíceps femoral e o bordo lateral da patela, sendo esta a referência para o pivô do goniômetro; no ponto central na proeminência óssea do trocânter maior, onde o centro da haste fixa deveria estar direcionado; e no centro do maléolo lateral, onde o centro da haste móvel deveria estar direcionado no final da avaliação (19). Os isquiostibiais foram avaliados através do Teste do Ângulo Poplíteo (TAP), com o participante em decúbito dorsal e quadril em $90^{\circ}$ de flexão, passivamente realizando extensão do joelho até o limite com o pé em posição neutra, sendo que a perna contralateral foi estabilizada com um cinto acima do joelho (20). 0 quadríceps-femoral foi avaliado através do Teste de Thomas (TT), em que o participante ficou em decúbito dorsal com os membros inferiores fora da maca, sendo realizada flexão de quadril e joelho da perna contralateral até que a coxa encostasse no tronco; utilizou-se uma faixa para estabilizar a perna ipsilateral acima do joelho (21). Os testes foram realizados três vezes, em cada membro, pelos mesmos avaliadores, sendo calculada a média para cada perna (Figura 1).

A metodologia de avaliação no dinamômetro segue o procedimento descrito por Kannus (22). Para determinar qual membro seria avaliado primeiro, foi realizado um sorteio com auxílio de uma moeda, de forma que o pesquisador responsável pela avaliação isocinética não soubesse qual era o MD dos participantes. Antes de cada avaliação, o dinamômetro isocinético foi calibrado, os sujeitos foram estabilizados com cintos, a angulação do encosto foi de $80^{\circ}$, a fossa poplítea ficou a três centímetros de distância do assento e o braço do isocinético foi preso a cinco centímetros acima do maléolo lateral. 0 joelho avaliado foi posicionado a $100^{\circ}$ da flexão $\left(0^{\circ}=\right.$ extensão completa) e o eixo de rotação do braço do dinamômetro foi alinhado com a parte lateral do côndilo femoral. A avaliação iniciou-se pela perna dominante; previamente à realização do teste, procederam-se três repetições submáximas de extensão/flexão do joelho para adaptação. Após essa etapa, foram realizadas cinco repetições utilizando contração concêntrica para extensão/flexão do joelho, com velocidade de $60^{\circ} / \mathrm{seg}$, para mensurar Pico de Torque (PT) e Trabalho Máximo (TM), e 15 repetições com velocidade angular de $240^{\circ}$ /seg, para Potência Máxima (PM), sendo que entre cada teste houve intervalo de dois minutos. Os mesmos procedimentos foram 


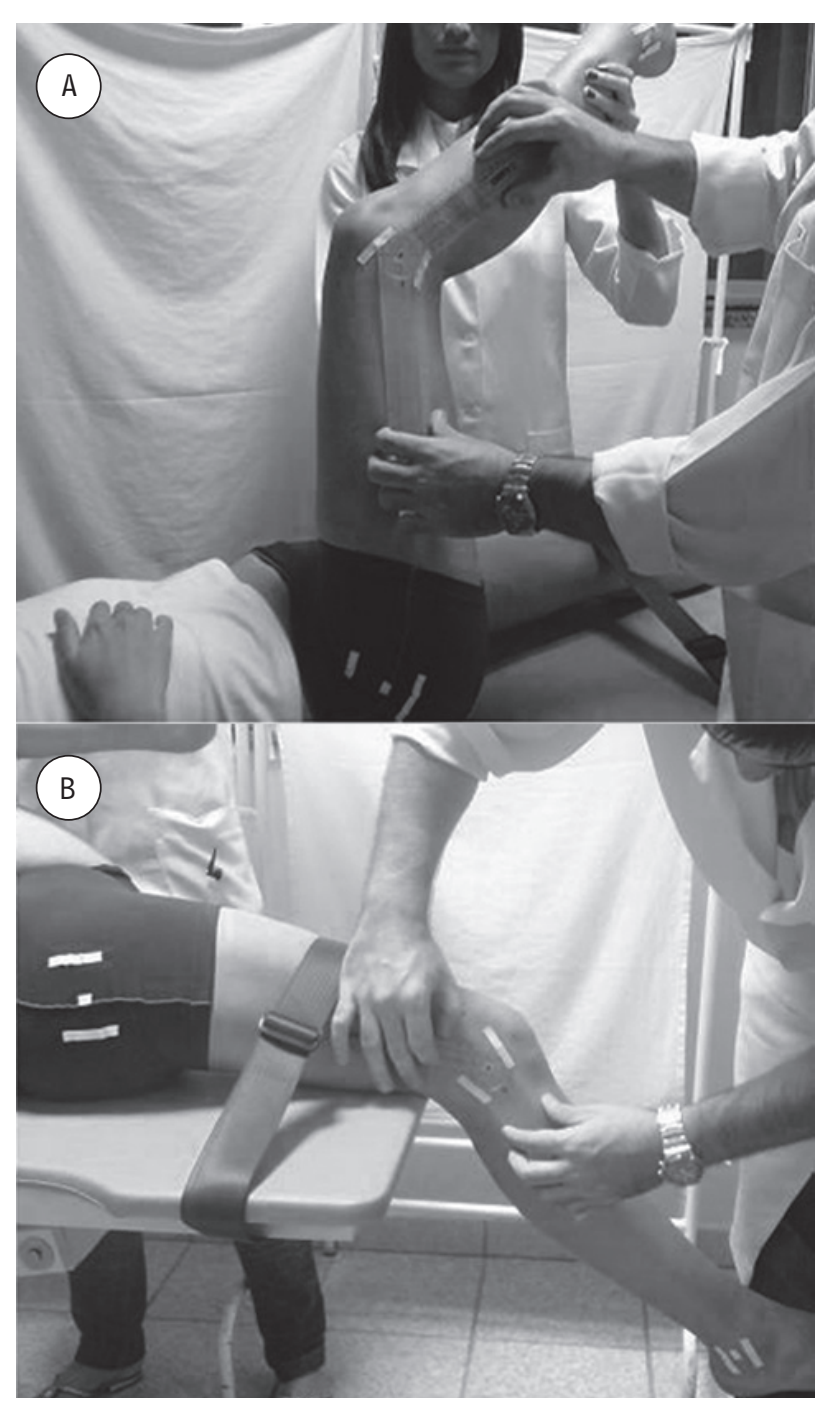

Figura 1 - A: Teste do ângulo poplíteo: membro a ser avaliado em $90^{\circ}$ de flexão do quadril, com extensão de joelho até o limite e perna contralateral estabilizada. B: Teste de Thomas: perna fora da maca, membro contralateral em flexão máxima em direção ao tronco, coxa homolateral estabilizada.

Fonte: Dados da pesquisa.

realizados no outro membro, e todas as avaliações foram realizadas pelo mesmo avaliador. Durante os testes, os participantes receberam estímulo verbal padronizado, para garantir o desempenho muscular máximo.

Para a presente pesquisa, foi considerado o melhor desempenho em cada variável isocinética estudada, e, para comparação do MD com o MND, foi utilizado o Índice de Simetria dos Membros (ISM), calculado pela divisão do MND pelo MD, multiplicado por 100 (MND/MD x 100) (23).
Para interpretação dos dados, utilizou-se um guia proposto por Sapega (24), que considera os seguintes critérios para comparação entre membros: quando o desequilíbrio for menor que $10 \%$, podem ser considerados normais; quando a diferença for entre $10-20 \%$, são possivelmente anormais; e aqueles com mais de $20 \%$ de diferença são provavelmente anormais.

\section{Análise estatística}

Avaliou-se, inicialmente, a condição de normalidade dos dados pelo teste de Kolmogorov-Smirnov. Após verificada a normalidade, utilizou-se o teste $\mathrm{t}$ para amostras pareadas, para comparar o MD e o MND quanto a flexibilidade (TAP e TT) e variáveis isocinéticas (PT, TM, PM). Para isso, utilizou-se o programa SPSS (Statistical Package for the Social Sciences v. 17.0), e a significância considerada no estudo foi de $\mathrm{p}<0,05$. A análise descritiva foi utilizada para variáveis antropométricas como idade, peso, altura e IMC.

\section{Resultados}

Dos 23 participantes da pesquisa, $20(86,9 \%)$ relataram o membro direito como sendo o dominante e três $(10,1 \%)$ relataram ser o membro esquerdo o dominante. Quanto à flexibilidade, não foram encontradas diferenças estatisticamente significantes, tanto no Teste do Ângulo Poplíteo como no Teste de Thomas $(\mathrm{p}>0,05)$ (Tabela 1).

Já quanto a Pico de Torque, Trabalho Máximo e Potência Máxima, tanto para extensão como para flexão do joelho, o membro dominante apresentou um desempenho significativamente superior quando comparado ao membro não dominante $(\mathrm{p}<0,001)$ (Tabela 1$)$. De acordo com o proposto por Sapega (24), determinando a diferença entre os membros de até $10 \%$ como normal, a minoria dos participantes atingiu deficits de acordo com a normalidade no PT dos extensores $(26,1 \%)$, no PT dos flexores (30,4\%), no TM dos extensores (17,3\%), no TM dos flexores (13\%), na PM dos extensores $(34,7 \%)$ e na PM dos Flexores (43,4\%) (Figura 2).

\section{Discussão}

As características do comportamento motor decorrentes da preferência lateral têm sido inves- 
Tabela 1 - Média e desvio-padrão dos membros dominante e não dominante quanto a flexibilidade e variáveis isocinéticas

\begin{tabular}{lcccc}
\hline & Dominante & Não dominante & Deficit & Valor de p \\
\hline TAP (graus) & $131,8 \pm 6,2$ & $132,6 \pm 6,6$ & $0,6 \%$ & 0,326 \\
TT (graus) & $108,6 \pm 9,1$ & $110,5 \pm 8,3$ & $1,7 \%$ & 0,338 \\
PT extensão (N.m) & $170 \pm 26,9$ & $144 \pm 26,6$ & $15,3 \%$ & $<0,001$ \\
PT flexão (N.m) & $95,5 \pm 20,5$ & $81,8 \pm 12,9$ & $14,4 \%$ & $<0,001$ \\
TM extensão (J) & $143,2 \pm 30,2$ & $117,7 \pm 24,2$ & $17,9 \%$ & $<0,001$ \\
TM flexão (J) & $92,4 \pm 26,3$ & $74,3 \pm 13,7$ & $19,6 \%$ & $<0,001$ \\
PM extensão (W) & $165,9 \pm 22,8$ & $144,6 \pm 32,9$ & $12,9 \%$ & $<0,001$ \\
PM flexão (W) & $129,9 \pm 35$ & $114,2 \pm 28,5$ & $12,1 \%$ & $<0,001$ \\
\hline
\end{tabular}

Fonte: Dados da pesquisa.

Legenda: TAP = teste do ângulo poplíteo; TT = teste de Thomas; PT = pico de torque; TM = trabalho máximo; PM = potência máxima.

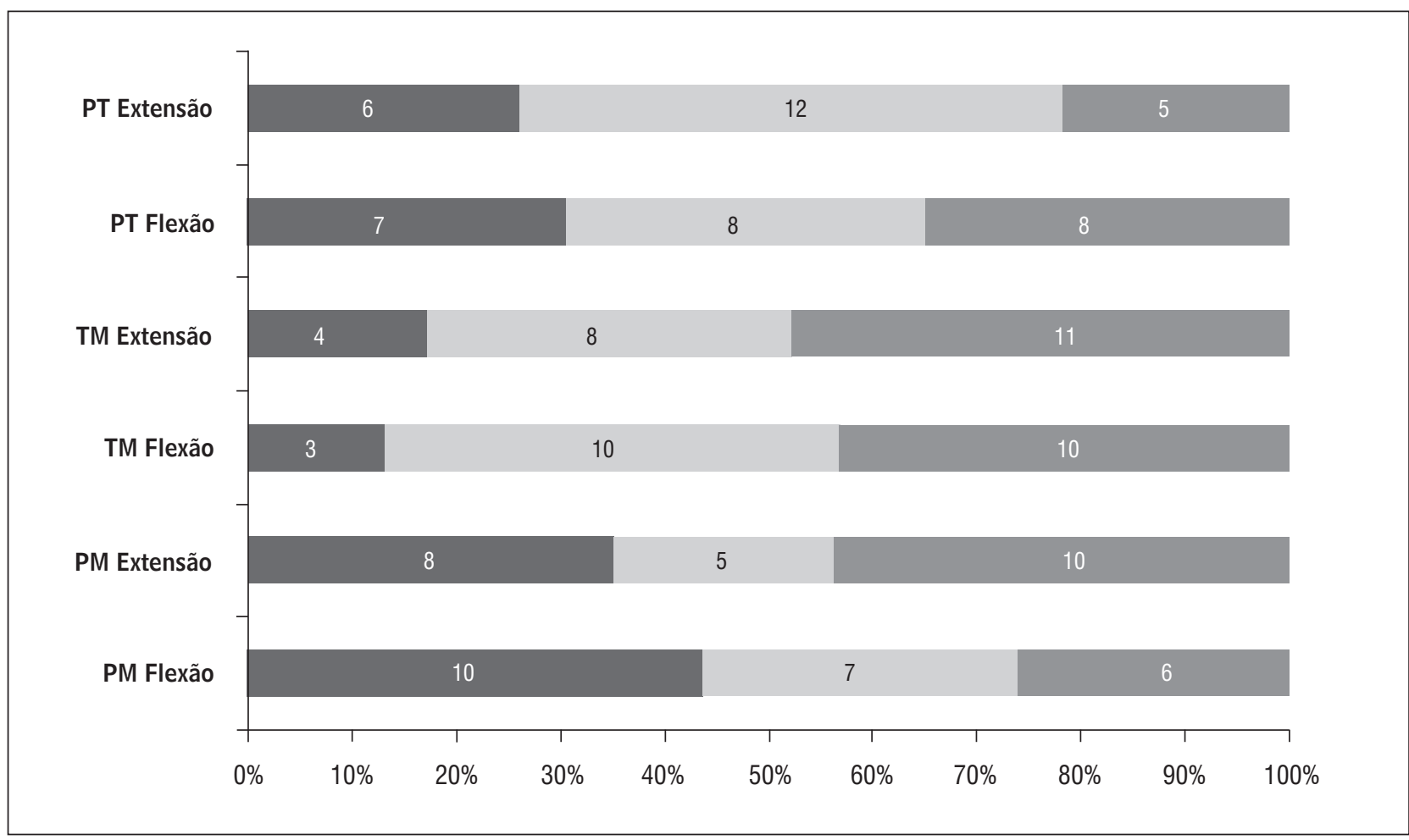

Figura 2 - Porcentagem correspondente à assimetria presente entre os membros nas variáveis isocinéticas avaliadas Fonte: Dados da pesquisa.

Legenda: PT: Pico de Torque; TM: Trabalho Máximo; PM: Potência Máxima.

tigadas por vários autores e de diferentes maneiras. Assimetrias laterais no comportamento motor humano estão presentes tanto em relação à preferência quanto ao desempenho $(5,8,9)$. Estudos utilizando ressonância magnética funcional tem claramente mostrado que a lateralização da atividade do cérebro existe e pode ser influenciada por vários fatores, incluindo a dominância dos membros
(2). Além disso, resultados de um estudo sugerem que o reflexo neural periférico durante o exercício poderia ser diferente entre o membro dominante e o não dominante (25).

Sadeghi et al. (3), em revisão da literatura, revelam que o comportamento assimétrico dos membros inferiores durante a deambulação sem deficiência foi abordado em várias pesquisas e que 
diferenças funcionais foram encontradas, sendo relacionadas à contribuição de cada membro na realização de tarefas de propulsão e de controle durante a caminhada. Essas diferenças podem levar a alterações na massa muscular e em propriedades contráteis (composição do tipo de fibra, arquitetura muscular) e/ou a diferenças na ativação neural máxima (26).

Um estudo verificando as diferenças de pressão plantar entre o MD e o MND em quatro movimentos relacionados ao futebol (corrida, "corte" para os lados, "corte" em 45으 e aterrissagem de um salto vertical) mostrou que a pressão plantar global foi maior no MD. Porém, especificando as fases dos quatros movimentos avaliados, a pressão plantar do MD foi maior na fase de decolagem, ao passo que a do MND foi maior na fase de aterrissagem. Os autores da pesquisa sugerem que o membro dominante tem tendência a desenvolver maior força e que o membro não dominante tem um papel mais importante na estabilização do corpo (27).

Na literatura, há muitas formas de avaliar comparativamente os membros inferiores, e os resultados são bastante controversos. Estudos têm utilizado o controle do equilíbrio estático (10, 18), o exercício de agachamento unilateral (11), a força isocinética de extensores do joelho e salto (28), os quais não são melhores quando executados com o membro dominante em relação ao não dominante. Esses achados questionam a utilidade e a importância de relatar a dominância da perna durante os testes no dinamômetro isocinético dos membros inferiores (29). Porém, as pesquisas comparativas de dominância unilateral privilegiam principalmente atletas e praticantes de atividades físicas, não podendo tomar esses resultados como padrão para populações não praticantes de exercícios físicos.

Nossos resultados indicam que o uso preferencial de um membro nas atividades diárias pode aumentar sua força em comparação ao contralateral. Em teoria, o membro dominante poderia ser mais treinado comparado ao não dominante, pelo efeito cumulativo a longo tempo das atividades diárias (30). De acordo com os resultados encontrados, em estudo comparando a força dos extensores do joelho de forma isométrica e isocinética, não houve diferenças significativas na contração voluntária máxima isométrica entre o MD e o MND; porém, nas velocidades de $60^{\circ}, 180^{\circ}$ e $300^{\circ} / \mathrm{seg}$, o MD apresentou força significativamente maior do que o MND (31). Utilizando testes mais funcionais, Van der Harst et al. (32) encontraram, no single-leg hop test, diferenças significantes entre o MD $(143 \pm 6,8 \mathrm{~cm})$ e o $\operatorname{MND}(136,8 \pm 5,7 \mathrm{~cm})$.

Hunter et al. (33) avaliaram 217 mulheres, com idades entre 20 e 89 anos, e verificaram um torque de extensão isométrica do joelho do lado dominante significativamente maior do que no membro não dominante. Dessa forma, deve-se ter cuidado ao utilizar o membro contralateral como fator determinante para avaliação e evolução do membro lesionado.

Rahnama et al. (5) consideraram, em sua pesquisa, um deficit de força contralateral quando o desequilíbrio excedeu $10 \%$ entre cada membro. $\mathrm{Na}$ presente pesquisa, considerando todas as variáveis isocinéticas avaliadas (PT, TM e PM de extensão e flexão do joelho), apenas $27,5 \%$ das avaliações tiveram diferenças de até $10 \%$, sendo que todas as outras apresentaram deficits maiores, chegando a diferenças bilaterais de $53 \%$ para $\mathrm{PT}, 53 \%$ para $\mathrm{TM}$ e $40 \%$ para PM.

Testes comparando os efeitos da preferência de um membro foram realizados em várias populações e utilizando diferentes avaliações. No tênis, a densidade mineral óssea e a força de preensão foram maiores no braço dominante (34). Em pesquisa com 41 jogadores de futebol conceituados, foi observado um deficit dos flexores do joelho do membro dominante, e um total de 28 jogadores apresentaram deficit maior que $10 \%$ entre os membros (5). Jacobs et al. (8) verificaram, em 42 indivíduos saudáveis, que o PT dos abdutores do quadril foi maior no MD quando comparado com o contralateral. De acordo com Lertwanich et al. (35), não se deve utilizar a força isocinética do membro superior contralateral para comparação com a evolução de um ombro possivelmente lesionado, já que, em indivíduos saudáveis, os movimentos de fisiológicos comparativos entre os ombros apresentaram diferenças de torque significativas.

Corroborando os estudos supracitados, quanto a PT, TM e PM de extensão e flexão do joelho, com mulheres assintomáticas e não praticantes de atividade física, elas apresentaram diferenças estatisticamente significantes (Tabela 1). Esses resultados indicam que a demanda diária no membro predileto 
causa melhor desempenho desse membro na avaliação isocinética.

Quanto à flexibilidade, MD e MND não apresentaram diferenças relevantes ( $p>0,05$ ), e esses resultados são similares a outros estudos. Macedo e Magee (36) analisaram a amplitude de movimento ativo e passivo de tornozelo, joelho, quadril, ombro, cotovelo e punho em 90 mulheres, usando um goniômetro padrão, e concluíram que algumas amplitudes apresentam diferenças quando comparadas com o lado contralateral, sendo clinicamente insignificantes. Rahnama et al. (5) avaliaram 41 jogadores de futebol e não identificaram diferenças quanto à flexibilidade entre os membros em relação à dominância.

\section{Conclusão}

A existência de desequilíbrios fisiológicos entre o membro dominante e o não dominante, no pico de torque, no trabalho máximo e na potência máxima de extensores e flexores do joelho, deve ser considerada na elaboração de medidas de prevenção em mulheres assintomáticas e não praticantes de atividade física. Com base nos resultados da pesquisa, verifica-se que indivíduos do sexo feminino podem desenvolver diferenças de performance entre os membros apenas com atividades do cotidiano.

As limitações deste estudo servem de sugestão para futuras pesquisas, com maior número de indivíduos, avaliação bilateral comparativa com diferentes populações, em diferentes articulações e com testes mais funcionais. Outros estudos também devem considerar os efeitos do período menstrual, tanto na flexibilidade quanto no desempenho muscular.

\section{Referências}

1. Gabbard C, Hart S. A question of foot dominance. J Gen Psychol. 1996;123(4):289-96. doi:10.1080/0022130 9.1996 .9921281$.

2. Barut C, Ozer CM, Sevinc O, Gumus M, Yunten Z. Relationship between hand and foot preferences. Int J Neurosci. 2007;117(2):177-85. doi:10.1080/00207450600582033.
3. Sadeghi H, Allard P, Prince F, Labelle H. Symmetry and limb dominance in able-bodied gait: a review. Gait Posture. 2000;12(1):34-45. doi:10.1016/ S0966-6362(00)00070-9.

4. Reiss M, Reiss G. Motor assymetry. Fortschr Neurol Psychiatr. 2000;68(2):70-9 doi:10.1055/s-2000-11648.

5. Rahnama N, Lees A, Bambaecichi E. Comparison of muscle strength and flexibility between the preferred and non-preferred leg in English soccer players. Ergonomics. 2005;48(11-14):1568-75. doi:10.1080/00140130500101585.

6. Zakas A. Bilateral isokinetic peak torque of quadriceps and hamstring muscles in professional soccer playes with dominance on one or both two sides. J Sports Med Phys Fitness. 2006;46(1):28-35. PMid:16596096.

7. Goble DJ, Marino GW, Potvin JR. The influence of horizontal velocity on interlimb in normal walking. Hum Mov Sci. 2003;22(3):271-83. doi:10.1016/ S0167-9457(03)00047-2.

8. Jacobs C, Uhl TL, Seeley M, Sterling W, Goodrich L. Strenght and fatigability of the dominant and nondominant hip abductors. J Athl Train. 2005;40(3):203-6. PMCid:1250264

9. Maupas E, Paysant J, Datie AM, Martinet N, André JM. Functional asymmetries of the lower limbs. A comparison between clinical assessment of laterality, isokinetic evaluation and electrogoniometric monitoring of knees during walking. Gait Posture. 2002;16(3):30412. doi:10.1016/S0966-6362(02)00020-6.

10. Hoffman M, Scrader J, Applegate T, Koceja D. Unilateral postural control of the functionally dominant and nondominant extremities of healthy subjects. J Athl Train. 1998;33(4):319-22. PMCid:1320581.

11. McCurdy K, Langford G. Comparison of unilateral squat strength between the dominant and non-dominant leg in men and women. J Sports Sci Med. 2005;4:153-9.

12. Tanaka T, Hashimoto N, Nakata M, Ito T, Ino S, Ifukube T. Analysis of toe pressure under the foot while dynamic standing on one foot in healthy subjects. J Orthop Sports Phys Ther. 1996;23(3):188-93. PMid:8919397.

13. Copranica L, Cama G, Fanton F, Tessitore A, Figura F. Force and power of preferred and non-preferred leg in young soccer players. J Sports Med Phys Fitness. 1992;32(4):358-63. PMid:1293418. 
14. Goldie PA, Bach TM, Evans OM. Force platform measurements for evaluating postural control: reliability and validity. Arch Phys Med Rehabil. 1989;70(7):510-7. PMid:2742465.

15. Kramer JF, Balsor BE. Lower extremity preference and knee extensor torques in intercollegiate soccer players. Can J Sport Sci. 1990;15(3):180-4. PMid:2257531.

16. Magalhães J, Oliveira J, Ascensão A, Soares J. Concentric quadríceps and hamstring isoknetic strength in volleyball and soccer players. J Sports Med Phys Fitness. 2004;44(2):119-25. PMid:15470308.

17. Carvalho T, Nóbrega AC, Lazzoli JK, Magni JR, Rezende L, Drummond FA, et al. Posição oficial da Sociedade Brasileira de Medicina do Esporte: atividade física e saúde. Rev Bras Med Esporte. 1996;2(4):79-81.

18. Lin WH, Liu YF, Hsieh CC, Lee AJ. Ankle eversion to inversion strength ratio and static balance control in the dominant and non-dominant limbs of young adults. J Sci Med Sport. 2009;12(1):42-9. doi:10.1016/j. jsams.2007.10.001.

19. Santos A. Diagnóstico clínico postural: um guia prático. São Paulo: Summus; 2001.

20. Ferreira GNT, Salmela LFT, Guimarães CQ. Gains in flexibility related to measures of muscular performance: impacto of flexibility on muscular performance. Clin J Sport Med. 2007;17(4):276-81. doi:10.1097/ JSM.0b013e3180f60b26.

21. Peeler J, Anderson JE. Effectiveness of static quadriceps stretching in individuals with patellofemoral joint pain. Clin J Sport Med. 2007;17(4):234-41. doi:10.1097/ JSM.0b013e3180f60afc.

22. Kannus P. Isokinetic evaluation of muscular performance: implications for muscle testing and rehabilitation. Int J Sports Med. 1994;15(1):11-8. doi:10.1055/s-2007-1021104.

23. Moksnes H, Risberg MA. Performance-based functional evaluation of non-operative and operative treatment after anterior cruciate ligament injury. Scand J Med Sci Sports. 2009;19(3):345-55. doi:10.1111/j.1600-0838.2008.00816.x.

24. Sapega AA. Muscle performance evaluation in orthopaedic practice. J Bone Joint Surg Am. 1990;72:156274. PMid:2254369.
25. Kapreli E, Athanasopoulos S, Papathanasiou M, Van Hecke P, Strimpakos N, Gouliamos A, et al. Lateralization of brain activity during lower limb joints movement. An fMRI study. Neuroimage. 2006;32(4):170921. doi:10.1016/j.neuroimage.2006.05.043.

26. Guette M, Gondim J, Martin A. Time-of-day effect on the torque and neuromuscular properties of dominant and non-dominant quadriceps femoris. Chronobiol Int. 2005;22(3):541-58. doi:10.1081/ CBI-200062407.

27. Wong P, Chamari K, Chaouachi A, Mao W, Wisloff U, Hong Y. Difference in plantar pressure between the preferred and non-preferred feet in four soccer-related movements. Br J Sports Med. 2007;41(2):84-92. doi:10.1136/bjsm.2006.030908.

28. Ostenberg A, Roos E, Ekdahl C, Roos H. Isokinetic knee extensor strength and functional performance in healthy female soccer players. Scand J Med Sci Sports. 1998;8(5 Pt 1):257-64. doi:10.1111/j.1600-0838.1998.tb00480.x.

29. de Ruiter CJ, de Korte A, Schreven S, de Haan A. Leg dominancy in relation to fast isometric torque production and squat junp height. Eur J Appl Physiol. 2010;108(2):247-55. doi:10.1007/ s00421-009-1209-0.

30. Minotti JR, Johnson EC, Hudson TL, Sibbit RR, Wise LE, Fukushima E, et al. Forearm metabolic asymmetry detected by 31P-NMR during submaximal exercise. J Appl Physiol. 67(1):324-9. PMid:2759961.

31. Hotta N, Yamamoto K, Sato K, Katayama K, Fukuoka Y, Ishida K. Ventilatory and circulatory responses at the onset of dominant and non-dominant limb exercise. Eur J Appl Physiol. 2007;101(3):347-58. doi:10.1007/s00421-007-0500-1.

32. Van Der Harst JJ, Gokeler A, Hof AL. Leg kinematics and kinetics in landing from a single-leg hop for distance. A comparison between dominant and non-dominant leg. Clin Biomech. 2007;22(6):674-80. doi:10.1016/j.clinbiomech.2007.02.007.

33. Hunter SK, Thompson MW, Adams RD. Relationships among age-associated strength changes and physical activity level, limb dominance, and muscle group in women. J Gerontol A Biol Sci Med Sci. 2000;55(6):26473. doi:10.1093/gerona/55.6.B264. 
34. Ducher G, Jaffre C, Arlettaz A, Benhamou CL, Courteix D. Effects of long-term tennis playing on the muscle-bone relationship in the dominant and nondominant forearms. Can J Appl Prhysiol. 2005;30(1):3-17. doi:10.1139/h05-101.

35. Lertwanich P, Lamsam C, Kulthanan T. Difference in isokinetic strength of the muscles around dominant and nondominant shoulders. J Med Assoc Thai. 2006;89(7):948-52. PMid:16881425.
36. Macedo LG, Magee DJ. Differences in range of motion between dominant and nondominant sides of upper and lower extremities. J Manipulative Physiol Ther. 2008;31(8):577-82. doi:10.1016/j.jmpt.2008.09.003.

Recebido: 02/09/2010

Received: 09/02/2010

Aprovado: 20/07/2012

Approved: 07/20/2012 